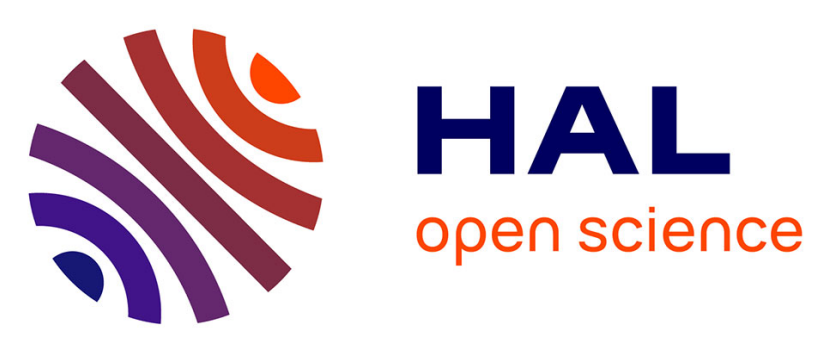

\title{
Endoluminal high-resolution MR imaging protocol for colon walls analysis in mouse model of colitis
}

Hugo Dorez, Raphaël Sablong, Laurence Canaple, Hervé Saint-Jalmes, Sophie Gaillard, Driffa Moussata, Olivier Beuf

\section{To cite this version:}

Hugo Dorez, Raphaël Sablong, Laurence Canaple, Hervé Saint-Jalmes, Sophie Gaillard, et al.. Endoluminal high-resolution MR imaging protocol for colon walls analysis in mouse model of colitis. Magnetic Resonance Materials in Physics, Biology and Medicine, 2016, 29 (4), pp.657-669. 10.1007/s10334016-0539-2 . hal-01296355

\section{HAL Id: hal-01296355 \\ https://hal.science/hal-01296355}

Submitted on 4 Apr 2016

HAL is a multi-disciplinary open access archive for the deposit and dissemination of scientific research documents, whether they are published or not. The documents may come from teaching and research institutions in France or abroad, or from public or private research centers.
L'archive ouverte pluridisciplinaire HAL, est destinée au dépôt et à la diffusion de documents scientifiques de niveau recherche, publiés ou non, émanant des établissements d'enseignement et de recherche français ou étrangers, des laboratoires publics ou privés. 


\section{Endoluminal high-resolution MR imaging protocol for colon walls analysis in mouse} model of colitis

Hugo Dorez ${ }^{1}$, Raphaël Sablong ${ }^{1}$, Laurence Canaple ${ }^{2}$, Hervé Saint-Jalmes ${ }^{3,4}$, Sophie Gaillard ${ }^{1}$, Driffa Moussata $^{1,5}$ and Olivier Beuf ${ }^{1}$

${ }^{1}$ Université de Lyon, CREATIS; CNRS UMR 5220; INSERM U1044; INSA-Lyon, Université Lyon 1, Villeurbanne, France

${ }^{2}$ Institut de Génomique Fonctionnelle de Lyon, Université de Lyon 1, UMR 5242 CNRS, Ecole Normale Supérieure de Lyon, Lyon, France

${ }^{3}$ LTSI; INSERM U642; Université Rennes 1, Rennes, France

${ }^{4}$ CRLCC; Centre Eugène Marquis, Rennes, France

${ }^{5}$ Hôpital Régional Universitaire de Tours - Service hépato-gastroentérologie, Tours, France

SHORT TITLE: Endoluminal MR imaging protocol for mouse colon walls exam

Corresponding author:

Olivier Beuf, $\mathrm{PhD}$

CREATIS, CNRS UMR 5220, Inserm U1044

Université Lyon 1, bât. 308 (ESCPE)

3 rue Victor Grignard

69616 Villeurbanne, FRANCE.

Tel: +33472431597

Fax: +33472448199 
26 WORD count of ABSTRACT $=200$

27 WORD count of TEXT $=6062$

28

$29 \quad$ Number of figures $=7$

30 Number of tables $=3$

$31 \quad$ Number of references $=53$

32

33

34

ACKNOWLEDGMENTS:

35

This work was supported by the LABEX PRIMES (ANR-11-LABX-0063) of "Université de 36 Lyon", within the program "Investissements d'Avenir" (ANR-11-IDEX-0007) operated by the French National Research Agency (ANR).

38

39 


\section{ABSTRACT:}

Object: An endoluminal MR imaging protocol including the design of an endoluminal coil (EC) was defined for high spatial resolution MR imaging of mice gastrointestinal walls at 4.7T.

Materials and Methods: A receive-only radiofrequency single-loop coil was developed for mice colon wall imaging. Combined with a specific protocol, the prototype was first characterized in vitro on phantoms and on vegetables. Signal-to-noise ratio (SNR) profiles were compared with a quadrature volume birdcage coil (QVBC). Endoluminal MR imaging protocol combined with the EC was assessed in vivo on mice.

Results: SNR measured close to the coil is significantly higher (10 times and up to $3 \mathrm{~mm}$ of the EC center) than the SNR measured with the QVBC. The gain in SNR can be used to reduce the in-plane pixel size up to $39 \times 39 \mu \mathrm{m}^{2}(234 \mu \mathrm{m}$ slice thickness) without time penalty. The different colon wall layers can only be distinguished on images acquired with the EC.

Conclusion: Dedicated EC provides suitable images for the assessment of mice colon wall layers. This proof of concept provides gain in spatial resolution and leads to adequate protocols, for the assessment of human colorectal cancer that can now be used as a new imaging tool for a better understanding of the pathology.

KEYWORDS: Colorectal Neoplasms, Magnetic Resonance Imaging, Colitis, Instrumentation 
INTRODUCTION:

63

Every year, on the overall cases of cancer diagnosed in the world, $13 \%$ are colorectal cancer (CRC) [1-3]. It is the second deadliest cancer in Western countriesjust behind lung cancer. The 5-years survival rate is higher than $80 \%$ when the cancer is diagnosed and treated at an early stage [4].The cancerogenesis of CRC has not been yet precisely established but two major pathways have been identified [5]:the chromosomal instability pathway (adenomacarcinoma sequence) and the microsatellite instability. Those pathways induce changes in the cellular proliferation with abnormal cells growing and infiltration into the gastrointestinal walls. The different stages of CRC infiltration are well known [6, 7].It is first characterized by an inflammation stage affecting the superficial layer of the colon wall. Then, the abnormal cells proliferation infiltrates the wall to deeper structures and, eventually, reaches the lymphatic system. Because the survival of patientsstrongly depends on the diagnosis stage, anearly and accurate diagnosisto improve thetherapeutic response efficacy is highly beneficial to the patient. Magnetic Resonance Imaging (MRI), computed tomography (CT), positron emission tomography (PET) andsome optical modalitiesprovide different kind of information when imaging gastrointestinal walls.However, it is still difficult (using those modalities and associated protocols) to assess fine structures from the abnormal cells proliferation.Today, endoscopic ultrasound (EUS) is widely used in clinical routine for the staging of colorectal cancer lesions [8-10]. It is an available and low costreal-time (motion free) imaging modality. Moreover, the sensitivity of EUS for rectal tumors invasion is approximately $88-95 \%$ while specificity is nearly 100\% (for stage 2 and more) [11]. However, EUS is especially used on rectal carcinomas and fewer studies have shown the potential of this modality on colon cancer [12-14]. EUS is also strongly dependent on operator and patient and thus require practice to perform anaccurate diagnosis. This is especially the case when examination is 
performed at early stages of CRC. Some studies describe the use of endoscopic MRI comparedto EUS [15-19] to assess colorectal region.MRI is well suited to investigate human soft tissues and provides a good spatial resolution for the characterization and staging of gastrointestinal lesions [20]. The modality provides ways of performing high-resolution quantitative maps, various contrast imaging and three-dimensional acquisitions but also performing magnetic resonance spectroscopy.However, colon and rectal walls assessment requires an even higher spatial resolution for visualizing different wall layers in detail [21]. It has been shown that spatial resolution in MRI could be used to depict more precisely, using EC, the gut wall than EUS,for N-staging [17] for example or for the staging of inflammation degrees and cancer lesions. Despite the fact that endoscopic MRI has demonstrated to be superior to MRI using external array coils, by providingexquisiteinformation of wall structures $[16,19,22,23]$, the use of endoscopic coils in human can induce strong local temperature increase at coil tip and along the coaxial cable [24-26]. While heating can be strongly reduced adding for example radiofrequency traps [24, 27], the use of inner coils on humans was currently limited to prostate or rectumand endoscopic MRI of colon based on the use of endoluminal coil is not performed on patient.Especially due to the Human colon anatomy (angle at the rectosigmoid junction) and theinvasive procedure (requires anesthesia and insertion of the coil into the patient's rectum).

Today, a better understanding of the CRC genesis to adapt therapeutics responses and increase the survival of patients is needed. This goes through the following of the adenomacarcinoma sequence(inflammation to cancer) on a model of CRC.The follow-up of this sequence on Human longitudinal studies based on endoscopic MRI is not yet possible due to current safety issues. Moreover, the development of CRC on Human is a long process that can last over decades to understand the growth mechanisms (inflammation evolving into cancer). Nevertheless, this can be doneon a mouse model of colitis [28], chemically induced by the 
combination of dextran sulfate sodium (DSS $2 \%$ in drinking water) and azoxymethan (AOM, $10 \mathrm{mg} / \mathrm{kg}$ ).Trying to understand the growth mechanisms of CRC and to assess the potential of MRI in a mouse model of colitis present several advantages: first, the pathology induced by the method introduced by Tanaka et al in 2003 is a reliable model with strong correlations between Human and mice CRC; then, it is possible to follow mice on a much shorter time period (typically six months); and, finally, the examination can be performed for each stages. This paper is describing an endoluminal MRI protocol dedicated to the examination of a mouse cancer model as a preliminary and mandatory step before to move to clinical Human CRC examinations.

While MRI is well suited to investigate abdominal-anatomical structures [20],colon and rectal walls assessment requires an even higher spatial resolution for visualizing different wall layersin detail [21], even more on mice (colon wall thickness $0.3-0.7 \mathrm{~mm}$ [29]), still within a limited acquisition time suitable for in vivo examination.

Endoluminal Coil (EC) is drastically increasing the signal-to-noise ratio (SNR) close to the coil compared to external array coil $[16,19,22,23]$. This kind of elongated loop, based on two coplanar copper traces, was already used for intravascular MRI and MRS in the midnineties [30]. The temperature around the EC was measured during in vitroacquisition [31]. No abnormal and critical temperature increasing was observed. EC can provide ahigher spatial and/or temporal resolution and image quality to improve the characterization and staging of colorectal lesions. The aim of this study was to develop, build and characterize an EC for mice rectal wall investigation together with the adapted protocol. The proof of concept of the whole protocol was demonstrated on a limited number of examinations at different stages of a mouse model of CRC. 
MATERIALS AND METHODS:

\section{Endoluminal Coil description:}

The EC was designed for mice colon investigation on a 4.7 T Bruker Biospec system (Bruker, Ettlingen, Germany). It is a single loop with dimensions sized to the colon mouse anatomy [32]which leads to a $30 \mathrm{~mm}$ length and a 1.6 mmouter diameter(heat shrink coating included). The copper tracksare $0.8 \mathrm{~mm}$ width and $0.8 \mathrm{~mm}$ thick with a copperthickness of $35 \mu \mathrm{m}$. Following these characteristics, the inductance and resistance of the copper tracks were calculated using the analytical expression of two-distanttraces equation.The coil loop and tuning and matching circuit (including a ground plane on the back side of the circuit)were mechanically carved (Circuit Board Plotters S63, LPKF Laser and Electronics®, Germany) on both sides of a FR4 epoxy substrate of $0.8 \mathrm{~mm}$ thickness (C.I.F®, Buc, France).Nonmagnetic electronic circuit components were soldered on a single face. The two longest conductors of the loop have been placedon each side of the substrate.A copper tape was used at the distal end of the coil to close the loop between the two faces (Fig1).One for connecting the loop to the upper face (straight arrows) and, the second one, to connect the direct current (DC) ground and radiofrequency (RF) signal ground together (dashed arrows)to the ground plane. This is done to avoid current loops between two different grounds. The RF signal was carried out by a coaxial cable (RG-58, Radiall®, France) and the DC current travels through a 8-wires cable (Alpha Wire ${ }^{\circledR}$, New Jersey, USA) connected on a nonmagnetic Fisher connector (Plugs 4032, Fisher Connectors ${ }^{\circledR}$, Suisse) to the coil. This latter decoupling cable is connected to the 14 pinsconnector of the Bruker decoupling box cable through a homemade Fisher-Bruker conversion cable.A heat shrink coating (PF3-135) houses the loop coil to isolate the tracks from colon and rectum tissues. The end of the coil was sealed with glue (Araldite PRECISION, Araldite ${ }^{\circledR}$, Bâle, Switzerland). When the EC is used,the 
electrical circuitis isolated with polytetrafluoroethylene (PTFE or Teflon, DuPont ${ }$, Wilmington, USA) to avoid contacts with tissues or liquids.

\section{Endoluminal Coil characterization:}

The resonant circuit is based on a RLC resonator circuit made of fixed nonmagnetic components (ATC, Huntington Station, New York, USA) and trimmer components (AT 57290, Temex Ceramics ${ }^{\circledR}$, Bordeaux, France) - Fig 1.Considering only the intrinsic parameters of the coil, which are the coil geometry, the materials used and the coupling effects (mutual inductance for example); the loop inductance and resistance wereestimated to $33.2 \mathrm{nH}$ and $0.1 \mathrm{Ohm}$ respectively. The values were calculated and corrected using measurements on a Vector Network Analyzer (E5070B - ENA Series, Agilent Technologies®, California, USA) - VNA.Intrinsic characteristics of electronic components (series resistances, self-inductances, parallel capacitances, self-frequency resonance) were modeled to simulate the precise electronic behavior of the coil. Modeling was done usinga Simulation Program with Integrated Circuit Emphasis(LTspiceIV, Linear Technology®, Milpitas, USA) feed with electronic components characteristics based on their individual datasheets. The value and manufacturers of the electronic components are summarized in table 1.

In thecoil design,there are basically four functionsto achieve.Briefly, the capacitor $C_{t}$ insures the tuning function, changing this value adjust the resonance frequency of the coil (on the S11 magnitude chart). Capacitors $C_{m, 1}$ and $C_{m, 2}$ are used for matching the coil to the $50 \mathrm{Ohms}$ impedance of receiver circuit in order to maximize the signal power transmission. $\operatorname{The} C_{B, 1}$ and $C_{B, 2}$ capacitors areused for blocking the DC current into theloop coil to improve the decoupling efficiency. It could alsocreate a local static magnetic field interfering with the static magnetic field $\mathrm{B}_{0}$. This ensures the correct decoupling of the loop coil. Mounted in 
series with $C_{t}$, capacitors can also contributemarginally for tuning the coil. Trimmer capacitors can be tuned mechanically with a nonmagnetic screwdriver to adjust the coil to match to $50 \mathrm{Ohms}$ at the working frequency of $200 \mathrm{MHz}$ (proton resonance frequency at 4.7 T). Finally, the decoupling circuit is represented by two Choke inductors and one PIN diode. The Choke inductors $L_{B, 1}$ and $L_{B, 2}$ protect the receiver amplifier from the induced RF current during the RF transmit pulses.

The PIN diode was mounted in parallel to the loop coil to minimize the series resistance and so maximizes the Quality factor (Q-factor) and thus the SNR [33, 34].The quality factor of the coil was measured using a Vector Network Analyzer (VNA). The loaded Q-factor $Q_{L}$ was measured with the EC inserted into a phantom containing a solution of saline solution and the unloaded Q-factor $Q_{0}$ was measured without the phantom.

\section{SNR assessment:}

All the experiments were performed on a 4.7 T Bruker Biospec System (Bruker, Ettlingen, Germany). In order to characterize the EC, each acquisition done with the EC was also compared to a ${ }^{1} \mathrm{H} 32 \mathrm{~mm}$ inner diameter quadrature volume birdcage coil (Rapid Biomedical ${ }^{\circledR}$, Rimpar, Germany) - QVBC dedicated to mouse body. However, the QVBC used does not include any decoupling circuit. As a consequence, the EC should not be inserted within the sample during QVBC operation. That is the reason why, when using the QVBC, an optical fiber (HCG 600, OFS®, Atlanta, USA) surrounded by a heat shrink coating was inserted into the mouse rectum instead of the EC. The outer diameter of the optical fiber and the heat shrink coating is $1.6 \mathrm{~mm}$. Then, when using the EC, the QVBC was replaced by a ${ }^{1} \mathrm{H}$ $72 \mathrm{~mm}$ inner diameter transmit linear volume coil (Rapid Biomedical@, Rimpar, Germany) VCincluding an active decoupling circuit. A protocol was developed and optimized for the purpose of EC assessment on phantom and small animals. Three axial 3D fast low-angle-shot 
(FLASH) sequences were used for anatomical and structure imaging but also for computing parametric T1 maps [37]. Then, one coronal FLASH 3D Slab sequence is performed to assess the colon along the EC. Finally, for computing parametric T2 maps, a 2D multiple spin-echo sequence was carried out. Table 2summarizes the sequences parameters used in the in vivoprotocol.

For SNR gain assessment, the coil was inserted into a12 $\mathrm{mm}$ outer diameter tube filled with a solution of $1.25 \mathrm{~g} / \mathrm{L}$ of $\mathrm{NiSO}_{4}$ and $5 \mathrm{~g} / \mathrm{L}$ of $\mathrm{NaCl}$ to mimic approximately the loading conditions found in livingtissues and have convenient relaxation times (T1-valueof $218 \mathrm{~ms}$ and T2-value of $140 \mathrm{~ms}$ ). First, the receiver EC was placed at the center of the VC used in transmit mode. The EC was driven by the active decoupling circuit during transmission. For SNR comparisons, axial images were acquired using FLASH 3D Slab sequences (table 3). Then, VC and EC were replaced by the QVBC and the optical fiber, respectively.With the same protocol, MR images were acquired using the QVBC(emission/reception mode). Slab was positioned manually to scan the same region in order to allow co-localization. The SNR were compared after correcting for difference in voxel size including the antialiasing steps, receive bandwidth and number of excitations between the EC and VCs [38].SNR profiles were normalized using thismethod and plotted in the same graph.

\section{Spatial resolution and contrast enhancement using an EC:}

The improvement of SNR provided by the EC can be used to decrease the voxel size without acquisition time penalties. To assess the spatial resolution achievable in the region of interest (ROI) with the EC within a reasonable acquisition time, a phantom containing the same $\mathrm{NiSO}_{4}$ solution was filled with optical fibers of standard diameters (ranging from $150 \mu \mathrm{m}$ to $2341000 \mu \mathrm{m})$. Using the transmit $\mathrm{VC}$ and the $\mathrm{EC}$ in reception mode, two sequences were acquired. First, an axial FLASH 3D Slab with a pixel size of $83 \times 83 \mu \mathrm{m}^{2}$ (slice of $234 \mu \mathrm{m}$ 
thick) was carried out(sequence 1 in table 3 ). Then, the sequence was adjusted to reduce the voxel size up to $39 \times 39 \mu \mathrm{m}^{2}$ keeping a slice thickness of $234 \mu \mathrm{m}$ (sequence 2 in table 3 ). The structures observed were measured using the software ImageJ [39] and compared to the optical fiber diameters measured with a digital caliper.

After having characterized the ECs on inorganic phantoms, experiments were carried out on organic phantoms such as onions in order to evaluate thesensitivity of such coils.Onions offer fine lamellar structures divided in several bulbs inside. The thickness of the different layers is, in average, the same as the one expected in mouse colon wall. Images performed on vegetables provide good markers to evaluate the sensitivity of the coil by analyzing the finest structure observable (for a given SNR).Experiments were performed using 3D Slab FLASH sequences $\left(20 \mathrm{~mm}\right.$ FOV, $25 \mathrm{~mm}$ slab thickness, TR/TE $=25 / 4.31 \mathrm{~ms}$ with $25^{\circ}$ flip angle, 256x192x96 matrix, $36764 \mathrm{~Hz}$ bandwidth and 11'31' acquisition time). The VC was turned in emission mode and the EC switched on reception mode.Finally, the spatial resolution was increased by reducing the pixel size down to $39 \times 39 \mu \mathrm{m}^{2}$ (10 mm FOV, $15 \mathrm{~mm}$ slab thickness, $\mathrm{TR} / \mathrm{TE}=20 / 6.1 \mathrm{~ms}$ with $20^{\circ}$ flip angle, $256 \times 256 \times 64$ matrix, $25 \mathrm{kHz}$ bandwidth and 10 '55" acquisition time) with a slice thickness equals to $234 \mu \mathrm{m}$.

\section{In vivomouse colon examination:}

In vivo experiments wereperformed to evaluate the feasibility and interest of using an EC in a mouse model of colitis. The study was carried out on mice chemically treated during two months to induce colitis using a combination of azoxymethan (AOM, intraperitoneal injection, $10 \mathrm{mg} / \mathrm{kg}$ body weight) and dextran sulfate sodium (DSS, in drinking water, concentration of $2 \%$ ) and four mice without treatment used as control.The experiments were in accordance with the rules and regulations of the UCBL Ethics Committee on animal experimentation. Prior to the examination, mice were kept on a 12 hours day/night rhythm in 
a $300 \mathrm{~cm}^{2}$ plastic cages with straw bedding, pellet food and tap water. Mice were anesthetized using an isoflurane tabletop station (TEM Sega ${ }^{\circledR}$, Lormont, France). Animals respiratory index was monitored during the experimentation by using a pressure sensor placed on the mouse chest. During the induction phase, mice were anesthetized with $3 \%$ of isoflurane and aspiration flow set up on $0.4 \mathrm{~L} / \mathrm{min}$. During imaging,the anesthesia was maintained with 1.4 to $1.7 \%$ isoflurane vaporization and aspiration flow set up on $0.4 \mathrm{~L} / \mathrm{min}$.Images of colon wall were first acquired by using thetransmit/receive QVBC. In that case, to mimic the presence of the EC, an optical fiber was carefully introduced into the mouse rectum using sterilized lubricant KY gel (K-Y®, Johnson \& Johnson, USA) and held in position with adhesive tape (strap on the MRI bed).Then, to assess colon wall layers with the EC, the QVBC was replaced by the linear VC. MR images were acquired to assess colon wall layers using described protocol in table 2 .

For each animals, T1- and T2-maps were computed using a linear fit from sequences acquired at different flip angle $\left(15^{\circ}, 20^{\circ}, 25^{\circ}\right)$ [37] and an exponential fit [40] at six different echo times $(12.8 \mathrm{~ms}, 25.21 \mathrm{~ms}, 37.81 \mathrm{~ms}, 50.42 \mathrm{~ms}, 63.03 \mathrm{~ms}$ and $75.63 \mathrm{~ms})$, respectively.Before the computation, images were threshold with an automatic method. The threshold level was set to three times the standard deviation measured in aROI with no signal (located in the top left corner of the image far from the EC).Two additional maps, representing the T1 linear fit quality and the T2 exponential fit quality, were also computed. To ensure reliable values of $\mathrm{T} 1$ and $\mathrm{T} 2$ relaxation times, fits having a coefficient of determination $\left(\mathrm{R}^{2}\right)$ inferior to 0.98 were systematically excluded from the representation.

For each in vitro and in vivo experiment, the coil was held in position with adhesive tape and not tilted during acquisitions. The EC was oriented in such a way to keep the $\mathrm{B}_{1}$ of the EC perpendicular to both static $\mathrm{B}_{0}$ and transmit $\mathrm{RF} \mathrm{B}_{1}$ magnetic fields. Hence maximum sensitivity and geometric decoupling are ensured respectively. As the colon natural behavior 
286 is to fold, the EC is always encompassed by colon structures ensuring almost uniform radial 287 signal intensity.

288 


\section{RESULTS:}

290 An endoluminal MR coil, fitting the constraints for in vivo experimentation on mice (colon

291

292

293 and rectum walls assessment) was designed and built. The prototypes are waterproof, reusable, biocompatible (with the investigated environment) and can be tuned/matched to the appropriate resonance frequency. The measured loaded Q-factor $\left(Q_{L}\right)$ of the coil was 32 and the coupling coefficient was found equal to 1.25 . This leads to a value of 72 for the unloaded Q-factor. The adaptation, at the 200.13 MHzworking frequency in loading conditions was 50.03 Ohms. The decoupling efficiency of the EC was tested using a VNA. When the pin diode is activated, the resonance frequency is shifted to $150 \mathrm{MHz}$ and the isolation at the resonance frequency reach $-43 \mathrm{~dB}$.

The EC provide a higher SNR in the close vicinity of the coil than the one obtained with quadrature volume birdcage coil. The EC geometry and dimensions fit with the use of the volume birdcage coil (72 $\mathrm{mm}$ inner diameter), without causing interferences and/or impacting the image quality. The absence of signal highlights in the close vicinity of the EC was confirmed when acquiring images with the body coil.Indeed, the continuously decoupled EC, not impacting the image around loop coil,was attested the correct functioning and efficacy of the decoupling circuit. The results of the SNR obtained with the EC compared with the QVBC acquired on phantom solutions are shown in figure 2.In receive mode, the SNR profile of the EC wasmuch higher than the one achieved with the QVBC up to 3 mmapart from the coil center. SNR profiles plotted in figure 2 werecorrected regarding the voxel size, bandwidth and number of excitations for a straightforward comparison. The EC SNRgain is 10 times greater at the close proximity and reaches the SNR of the QVBC at approximately $3 \mathrm{~mm}$ from the center.

A precise measurement of the structures observable in the loop-coil field of view was achieved on the phantomconstituted with calibrated optical fibers (figure 3).Optical 
fibersdiameters were measured with a standard deviation of $\pm 6.3 \mu$ musing the ImageJ software and then compared with a digital caliper (nominal precision $\pm 10 \mu \mathrm{m}$ ).

Experiments led on vegetables have shown the feasibility to obtain high spatial resolution images and depict structural details. It is possible to distinguish the lamellar structures of the onion to approximately 3 mmof the coil center (figure 4). White arrows on figure 4localizethe same thin slice of the onion obtained with the VC (figure 4a) and with the EC (figure $4 \mathrm{~b}$ and 4c) at two different spatial resolutions (different SNR).

\section{In vivo Experiments}

The EC geometry fits the mouse anatomy and is adapted to in vivo experiments. Once the mouse is anesthetized, the insertion of the EC performed by an experimented gastroenterologist is simple and harmless for the animal. The animal's recovery time is short and no signs of prostration and/or pain were observed during the following few hours in accordance with the French legislation. Images acquired in vivo show a very good quality (figure 5), in term ofcontrast and high spatial resolution. The use of the QVBC does not allow a correct visualization of colon walls due to limited achievable spatial resolution. Whereas the SNR gain provided by the EC is used to increase spatial resolution and to depict nicely the mouse rectum wall layers and surrounding structures.On figure 5b, the map ratio of $\mathrm{SNR}_{\mathrm{EC}} / \mathrm{SNR}_{\mathrm{QVBC}}$ is overlaid on MR images and undoubtedly illustrates the improved $\mathrm{SNR}$ area given by the EC. The white arrow on figure 5a1 and 5c locates a blood vessel used for co-localization. The examination with the EC gives a better visualization of colorectal wall layers and surrounding structures (figure $5 \mathrm{~d}$ and $5 \mathrm{e}$ ). White arrows locate also the mucosasubmucosa complex. Using such a coil allows to increase spatial resolution up to $39 \mu \mathrm{m}$ inplane resolution (with $234 \mu \mathrm{m}$ slice thickness, see figure $6 \mathrm{a}$ and $6 \mathrm{~b}$ ). It is then possible to well 
338 distinguish the mucosa complex in details. (1) is the mucosa, (2) the submucosa and (3) the 339 muscularis externa. On figure 6b,(4) locates a thin muscle layer.

340 The T1- and T2-values of structures around the EC can be seen on figure 7.The maps have 341 been calculated and plotted with an estimation of the fit quality to ensure reliable values.It is 342 worth noting that this parametric procedure removes the strong signal intensity decay that 343 could lead to misinterpretation on the magnitude images. T1- and T2-maps plotted from two 344 different slices illustrate different structures; (1) identifies a muscle structure and (2) the colon 345 wall. The averaged $\mathrm{T} 1$ relaxation times were measured at $800 \mathrm{~ms} \pm 100 \mathrm{~ms}$ and $3461200 \mathrm{~ms} \pm 200 \mathrm{~ms}$ respectively.Then, looking at the T2 maps, mucosa layer and musclaris 347 externa are clearly differentiable (3) and (4)on figure 7).The T2 relaxation times are nearly 348 identical, $80 \mathrm{~ms} \pm 10 \mathrm{~ms}$ for both structures. Finally, $*$ locates the submucosa which tends to 349 have lower $\mathrm{T} 2$ values $(50 \mathrm{~ms} \pm 10 \mathrm{~ms})$. The fit quality maps show a reproducible method for 350 T1- and T2-values with only a few points excluded in the field-of-view considered. 


\section{DISCUSSION:}

EC have been used since the late eighties [41] but essentially for visualizing human prostate

355 and rectum regions [42-45].Currently, the major problem lies in the heat induce by the RF current into conductors. Several studies have shown the potential of using RF traps to reduce this increase in temperature $[24,26,46]$. Optical transmission and optical decoupling system have also been proposed in order to replace conventional coaxial cable [47, 48]. More recently, the exploration of metamaterials have open new perspectives for alternative ways of RF signal transmission [49]. These metamaterials could overcome several coaxial cable disadvantages such as high propagation loss or heating along the conductor in the presence of electrical fields. But also, reaching the Human colon is difficult due to the $90^{\circ}$ angle and assessing higher structures in the colon may require flexible endorectal MR probes. Other studies have shown the high potential of using ECson preclinical models such as pigs [50], rabbits [16, 19] and rats [15]. But, it is the first reported study assessing colon and rectum walls using dedicated endorectal coils on a mouse model of colitis (inflammation evolving into cancer).

In this study, the feasibility to design an EC for small animal colon wall imaging such as mice was demonstrated. It has been shown that EC can provide typical $40 \mu \mathrm{m}$ in-plane pixel images with a slice thickness of $234 \mu \mathrm{m}$ along digestive tract.External volume coils provide less detailed images in similar conditions (same sequence parameters and position). It enables the visualization of colon wall layers and deeper structures with a spatial resolution and SNR not achievable with quadrature volume birdcage coil dedicated to mice gastrointestinal imaging. For decoupling the EC during the emission of radiofrequencies RF pulses, a parallel decoupling circuit was used. This choice was made to decrease the total resistance of the coil. Indeed,regarding the coil's dimensions and components, one third to one half of the total 
circuit resistance is due to the PIN diode characteristics alone.Placing the PIN diode in series leads in higher decoupling efficiency but decreases the quality factor and the SNR as well.

379 When the PIN diode is placed in parallel of the tuning capacitor, the resistance is lowered. The quality factor of the coil is improvedbut the decoupling efficiency is reduced. The PIN diode is not entirely shutting down the loop coil and a weak fraction of the radiofrequency

382 signal can still go through into the loop.In principle a coil having one or two parallel PIN diodes mounted in series with the loop would benefit from a better decoupling performance. Even if this solution is feasible for in vitro experiments it is not compatible with in vivo experiments.PIN diode used here are bigger components than case A ATC capacitors. Several PIN diodes on the coil will result in blocking the access to trimmer capacitors.

Using those trimmer capacitors help for tuning/matching the coil at the desired frequency but this kind of component has a poor life time, due to their mechanism working with a screwdriver, and had to be changed regularly. Usually, trimmer capacitors are linked to insulating sticks for tuning and matching. In the case of small coils it is difficult to have such a configuration.

A heat shrink coating covers the EC duringin vivo or in vitro experiments to prevent the sensor from immediate short-circuit risk. Consequently,the copper tracks are isolated from the surrounding mediumbut this protection layer weakens the maximum SNR achievable in the close vicinity of the coil. Using a thinner protection could improve the SNR close to the coil.Besides, the EC is wrapped with PTFE to protect the electronic circuit during in vivo 397 experiments:as the coil is used in wet environment, long-term oxidation of the copper tracks 398 can appear if the PTFE is not removed after each use and the EC not properly dried. 399 Oxidation could lead to short-circuits, deterioration of the copper tracks and/or components 400 and degradation of transmission line properties.A protective plastic cover will be designed 401 and printed to improve protection. SNR comparisons could not be achieved using the QVBC 
at the exact same position due to the absence of decoupling circuit on this coil. The comparison was then done sequentially slice by slice and correlated with images acquired with the EC. Mouse's body examinationwould hardly be done with the available $70 \mathrm{~mm}$ inner diameter birdcage coil. However, the use of a $40 \mathrm{~mm}$ inner diameter linear birdcage coil with active decoupling system would easily allow switching between EC and volume coil. Beside the easier and straightforward comparison in SNR, this setup would allow to acquire very local images of the colon as well as more regional images of the mouse. This setup would be a considerable way of improvement.

The phantom containing optical fibers was useful to assess the size of structures that could be observed (from $150 \mu \mathrm{m}$ to $1 \mathrm{~mm}$ ) in the EC FOV. On the figure 3, optical fibers seem to be equally distributed around the EC.Asoptical fibersfell downto the bottom of the container the upper part of the phantom was not containing enough fibers. More of them will have to be added to distribute correctly optical fibers around the sensor.Despite the fact that the sequences choose for imaging the phantom had a slice thickness of $234 \mu \mathrm{m}$, no partial volume effect was observe when measuring outer diameter of the optical fibers.

For the experimentations done on vegetables, onions have been chosen for their structure. Despite the fact that onions present very thin lamellar patterns, it can be assimilated to a semirigid material. Themolecules of water are constrained by their environment. So, when looking closely to the lamellar structure of this vegetable, it appears that air gaps are located between each slice. This causes some susceptibility artefacts. Moreover, the slice position chosen for the comparison wasnot exactly the same than the one obtain with the VC.To be able to localize the same structures between body coil images and EC images, the VC was used instead of the QVBC. The SNR of the VC allows the overall visualization of several layers of the vegetable. But to depict lamellar structure, the $\mathrm{VC}$ is inappropriate due to insufficient SNR. The use of the EC increases drastically the local SNR and fine structures can be 
visualized. But it also causes a highlight on the image, in the close vicinity of the coil. To

428 obtain the same details with the QVBC, the same sequence was done using six averages; the acquisition time was increase to $1 \mathrm{~h} 15 \mathrm{~min}$.

In vivo acquisitions show a huge improvement in the differentiation of colon wall layerscomparedto the QVBC. The designed EC fits with the straight configuration of the mouse colon. Indeed, human colon presents curves which cannot be passed using such a rigid coil. For one of the healthy mouse, the colon was sampled for further analyzes.During the dissection, the length, average diameter and thickness of the colon were approximately measured, using a digital caliper, to optimize the coil loop.

The protocol duration developed for the need of the study wasshort enough ( $38 \mathrm{~min})$ to ensure the safe examination and recovery of the animal. It provides anatomical and parametric images (T1 and T2 values). Three scout sequences with orthogonal plane orientationswere first done with the VC in emission/reception mode. Acquisitions were compared between images acquired with and without a trigger deviceusing a respiratory sensor place on the lower part of the abdomen. Though respiratory movement is ample and cardiac beats are rapid, those movements are far from the colon and no significant differences were observed.

443 So, no sequence triggering was performed in order to reduce the acquisition time. Nevertheless, colon can be affected by peristaltic movements. These movements have been evaluated using an optical system developed for this purpose. Movements of colon walls are slow, not regular and limited in amplitude.The degradation induced by this motion on image 447 quality is thus difficult to evaluate.A further analysis will be done to quantify this movement 448 and see the influence on MR images.

449 The EC provides a high SNR in its close vicinity but the SNR drops down heavily. Up to 4503 mmfrom the center, the SNR is higher than the one with the QVBC, further the sensitivity 451 of the EC begins very poor. The colon wall thickness was measuresin the range of 
0.2 to $0.5 \mathrm{~mm}(0.3-1 \mathrm{~mm}$ in the literature $[28,51])$. Regions of interest, such as colon walls, are closely located to the loop coil where the EC provides a very high SNR.Looking further the two first millimeters is not required for the purpose of this study.On figure 6, thein-plane pixel size was reduced $(\sim 39 \mu \mathrm{m}$ pixel width with a slice thickness of $234 \mu \mathrm{m})$. As the SNR is decreasing with the improvement of spatial resolution, the gain in SNR provided by EC is used to define the best compromise between pixel size and signal intensity. As the QVBC does not possess a decoupling circuit, the colocalization was done using anatomical markers. Blood vessels are convenient to localize the slice position. The mouse bladder and vertebrae can also be used as convenient landmarks.

Even if an optical endoscopic control exam is realized before inserting the coil into the mouse rectum, sometimes the lumen is still filled with feces and other natural substances. Those feces tend to drastically decrease the signal leading in an important loss of contrast around the feces. The systematic endoscopic control combined with a medication controlling the peristaltic movement (loperamide for example) could help to reduce the presence of feces in the lumen.During the evolution of the pathology, bleeding can also appear. When the EC is pulled out from the colon the presence of blood is sometimes noticed. This also induces distortion into the image quality and has to be taken into account for image processing.

Computed parametric T1- and T2-maps remove the strong SNR decay associated with the use of the EC. It is a fact that these strong intensity variations reduce the way the image is ridden compare to uniform signal intensity (provided by a QVBC for example).The T1 and T2 relaxation times obtained on the colon walls present an important standard deviation from the mean value. This is due to heterogeneity and size of the in vivo structures but also depends on the fit quality. Theoretically, in each pixel it is possible to obtain a value of the T1 and T2. Practically, some fits present a coefficient of determination to low to be taken into account. A part of the problem is due to the high and rapid variation of structures observed in vivo. 
Moreover, structures are very thin and can be essentially made of fat (for deeper structures for example). The non-significant values are removed from the results and can cause holes in the maps. A further study will beto evaluate the influence of the number and values of flip angles on fit quality. The 0.98 threshold here is chosen following what anatomical structures need to be seen in the EC FOV. It corresponds to a setting neither too general nor drastic. Information furnish by a fit quality of 0.98 are corresponding to the colon wall and surrounding structures. When designing the coil, there were no considerations of heating and/or specific absorption rate (SAR) taken into account.Indeed, during MR procedures, the radiofrequency power transmitted (from the emission coil) for imaging is turned into heat through resistive losses. Furthermore, when a conductor is placed within the imaging volume (as with the use of ECs) the RF electrical field accompanying the RF magnetic field pulses induces currents that can causelocal concentration of the SAR $[25,26,52,53]$.In our case, the loop can be considered as a wire but the quarter wavelength (at $200 \mathrm{MHz}$ ) is much higher than the length of the loop. It has been assumed that wire lengths less than a quarter wavelengths are generally safe. Thepresence of an active decoupling circuit is also mandatory toreduce SAR concentration [24].Further work will be to combine the EC with magnetic resonance spectroscopy (MRS) in the colon wall. MRS could provide information on the biochemical composition of deep tissue layers. Quantitative analysis of the biochemical contents could also bring new markers to improve the measurements. But several challenges still lie ahead, such as strong $\mathrm{B}_{0}$ inhomogeneities and phase shift due to the difference of magnetic susceptibility between air in the lumen and wall tissues.

In order to exploit the described work, a longitudinal follow up over six months involving 24 mice will be performed to explore in vivo the gastrointestinal wall in its entire depth. MR ECs open new perspectives as a minimally invasive technique to characterize colorectal 
501 cancer lesions and inflammatory bowel disease and then, offer a new technique to better 502 understand the development of the colon wall pathologies.

503 
504 CONCLUSION:

505 In conclusion, an endoluminal coil was developed for imaging the colon and rectum walls on 506 a mouse model of colitis. The strong local SNR increase provided by the use of such coils 507 allows imaging the gastrointestinal tract at very high spatial resolution (up to approximately $50840 \mu \mathrm{m}$ in plane resolution with a slice thickness of $234 \mu \mathrm{m})$. Associated to a dedicated 509 protocol, it provides details in deep still thin structures of colon wall.

510 


\section{Author's Contribution:}

512

513 DOREZ: Protocol/project development, data collection or management and data analysis

514 SABLONG: Protocol/Project development, data collection

515 CANAPLE: Protocol/project development

516 SAINT-JALMES: Protocol/project development

517 GAILLARD: Data collection

518 MOUSSATA: Protocol/project development, data collection or management

519 BEUF: Protocol/project development, data collection or management

520 


\section{COMPLIANCE WITH ETHICAL STANDARDS:}

522 Disclosure of potential conflicts of interest: the authors declare that they have no conflict of 523 interest.

524

525 Research involving animals: all applicable international, national, and/or institutional 526 guidelines for the care and use of animals were followed.

527 All procedures performed in studies involving animals were in accordance with the ethical 528 standards of the institution or practice at which the studies were conducted.

529 


\section{REFERENCES}

1. Bray F, Jemal A, Grey N, Ferlay J, Forman D (2012) Global cancer transitions according to the Human Development Index (2008-2030): a population-based study. Lancet Oncol 13:790801.

2. Jemal A, Bray F, Center MM, Ferlay J, Ward E, Forman D (2011) Global cancer statistics. CA Cancer J Clin 61:69-90.

3. Siegel R, DeSantis C, Jemal A (2014) Colorectal cancer statistics, 2014. CA Cancer J Clin 64:104-117.

4. O'Connell JB, Maggard MA, Ko CY (2004) Colon Cancer Survival Rates With the New American Joint Committee on Cancer Sixth Edition Staging. J Natl Cancer Inst 96:1420-1425.

5. Takayama T, Miyanishi K, Hayashi T, Sato Y, Niitsu Y (2006) Colorectal cancer: genetics of development and metastasis. J Gastroenterol 41:185-192.

6. Bisognin A, Pizzini S, Perilli L, Esposito G, Mocellin S, Nitti D, Zanovello P, Bortoluzzi S, Mandruzzato $S$ (2014) An integrative framework identifies alternative splicing events in colorectal cancer development. Mol Oncol 8:129-141.

7. Fearon ER (2011) Molecular Genetics of Colorectal Cancer. Annu Rev Pathol Mech Dis 6:479-507.

8. Vanagunas A, Lin DE, Stryker SJ (2004) Accuracy of Endoscopic Ultrasound for Restaging Rectal Cancer Following Neoadjuvant Chemoradiation Therapy. Am J Gastroenterol 99:109-112.

9. Puli SR, Reddy JBK, Bechtold ML, Choudhary A, Antillon MR, Brugge WR (2009) Accuracy of Endoscopic Ultrasound to Diagnose Nodal Invasion by Rectal Cancers: A Meta-Analysis and Systematic Review. Ann Surg Oncol 16:1255-1265.

10. Puli SR, Bechtold ML, Reddy JBK, Choudhary A, Antillon MR (2010) Can Endoscopic Ultrasound Predict Early Rectal Cancers That Can Be Resected Endoscopically? A Meta-Analysis and Systematic Review. Dig Dis Sci 55:1221-1229.

11. Puli SR, Bechtold ML, Reddy JBK, Choudhary A, Antillon MR, Brugge WR (2009) How Good is Endoscopic Ultrasound in Differentiating Various T Stages of Rectal Cancer? Meta-Analysis and Systematic Review. Ann Surg Oncol 16:254-265.

12. Vander Noot MR, Eloubeidi MA, Chen VK, Eltoum I, Jhala D, Jhala N, Syed S, Chhieng DC (2004) Diagnosis of gastrointestinal tract lesions by endoscopic ultrasound-guided fine-needle aspiration biopsy. Cancer 102:157-163.

13. Hurlstone DP, Brown S, Cross SS, Shorthouse AJ, Sanders DS (2005) Endoscopic Ultrasound Miniprobe Staging of Colorectal Cancer: Can Management Be Modified? Endoscopy 37:710714.

14. Hurlstone DP, Brown S, Cross SS, Shorthouse AJ, Sanders DS (2005) High magnification chromoscopic colonoscopy or high frequency $20 \mathrm{MHz}$ mini probe endoscopic ultrasound staging for early colorectal neoplasia: a comparative prospective analysis. Gut 54:1585-1589.

15. Pilleul F, Beuf O, Armenean M, Scoazec JY, Valette PJ, Saint-Jalmes H (2004) In vitro rat colonic wall imaging with MR endoluminal coil: Feasibility study and histologic correlations1. Acad Radiol 11:795-801. 
16. Beuf O, Pilleul F, Armenean M, Hadour G, Saint-Jalmes H (2004) In vivo colon wall imaging using endoluminal coils: Feasibility study on rabbits. J Magn Reson Imaging 20:90-96.

17. Maldjian C, Smith R, Kilger A, Schnall M, Ginsberg G, Kochman M (2000) Endorectal surface coil MR imaging as a staging technique for rectal carcinoma: a comparison study to rectal endosonography. Abdom Imaging 25:75-80.

18. Syms R, Young I, Wadsworth C, Taylor-Robinson S, Rea M (2013) Magnetic Resonance Imaging Duodenoscope. IEEE Trans Biomed Eng 60:3458-3467.

19. Pilleul F, Beuf O, Godefroy C, Scoazec J-Y, Armenean M, Armenean C, Perrin E, Valette P-J, Jalmes HS (2005) High-resolution MR imaging appearance of colonic tissue in rabbits using an endoluminal coil. Magn Reson Mater Phy 18:238-244.

20. Klessen C, Rogalla P, Taupitz M (2007) Local staging of rectal cancer: the current role of MRI. Eur Radiol 17:379-389.

21. Beaumont C, Pandey T, Gaines Fricke R, Laryea J, Jambhekar K (2013) MR Evaluation of Rectal Cancer: Current Concepts. Curr Probl Diagn Radiol 42:99-112.

22. Armenean M, Beuf O, Pilleul F, Saint-Jalmes H (2001) Endoluminal loop radiofrequency coils for gastrointestinal wall imaging. Proc. 23rd Annu. Int. Conf. IEEE Eng. Med. Biol. Soc. pp 30523055

23. Armenean M, Beuf O, Pilleul F, Saint-Jalmes H (2004) Optimization of endoluminal loop radiofrequency coils for gastrointestinal wall MR imaging. IEEE Sens J 4:57-64.

24. Ladd ME, Quick HH (2000) Reduction of resonant RF heating in intravascular catheters using coaxial chokes. Magn Reson Med 43:615-619.

25. Nitz WR, Oppelt A, Renz W, Manke C, Lenhart M, Link J (2001) On the heating of linear conductive structures as guide wires and catheters in interventional MRI. J Magn Reson Imaging 13:105114.

26. Shellock FG (2000) Radiofrequency Energy-Induced Heating During MR Procedures: A Review. J Magn Reson Imaging 12:30-36.

27. Gauss R, Wong E (2009) RF traps for radio frequency coils used in MRI. U.S. Patent No. 7,622,928.

28. Tanaka T, Kohno H, Suzuki R, Yamada Y, Sugie S, Mori H (2003) A novel inflammation-related mouse colon carcinogenesis model induced by azoxymethane and dextran sodium sulfate. Cancer Sci 94:965-973.

29. Aychek T, Vandoorne K, Brenner O, Jung S, Neeman M (2011) Quantitative analysis of intravenously administered contrast media reveals changes in vascular barrier functions in a murine colitis model. Magn Reson Med 66:235-243.

30. Atalar E, Bottomley PA, Ocali O, Correia LC, Kelemen MD, Lima JA, Zerhouni EA (1996) High resolution intravascular MRI and MRS by using a catheter receiver coil. Magn Reson Med 36:596-605.

31. Verret JM, Pilleul F, Rabrait C, Beuf O (2012) RF heating reduction associated to an MR endoluminal coil at 3T. ESMRMB 2012 29th Annu. Sci. Meet. Toulouse, p 143 
32. Komárek V (2012) Chapter 2.2 - Gross Anatomy. In: Hedrich HJ (ed) Lab. Mouse Second Ed. Academic Press, Boston, pp 145-159

33. Doty FD, Entzminger G, Kulkarni J, Pamarthy K, Staab JP (2007) Radio frequency coil technology for small-animal MRI. NMR Biomed 20:304-325.

34. Hoult DI, Richards RE (1976) The signal-to-noise ratio of the nuclear magnetic resonance experiment. J Magn Reson 24:71-85.

35. Kajfez D, Hwan EJ (1984) Q-Factor Measurement with Network Analyzer. IEEE Trans Microw Theory Tech 32:666-670.

36. Ginzton EL (1958) Microwave Q Measurements in the Presence of Coupling Losses. IRE Trans Microw Theory Tech 6:383-389.

37. Cheng H-LM, Wright GA (2006) Rapid high-resolution T1 mapping by variable flip angles: Accurate and precise measurements in the presence of radiofrequency field inhomogeneity. Magn Reson Med 55:566-574.

38. Hashemi RH, Bradley WG, Lisanti CJ (2012) MRI: The Basics. Lippincott Williams \& Wilkins

39. Rasband WS, et al. (1997) \& ImageJ. Bethesda, Md, USA

40. Gibbs P, Tozer DJ, Liney GP, Turnbull LW (2001) Comparison of quantitative T2 mapping and diffusion-weighted imaging in the normal and pathologic prostate. Magn Reson Med 46:1054-1058.

41. Schnall MD, Lenkinski RE, Pollack HM, Imai Y, Kressel HY (1989) Prostate: MR imaging with an endorectal surface coil. Radiology 172:570-574.

42. Stoker J, Rociu E (1999) Endoluminal MR imaging of diseases of the anus and rectum. Semin Ultrasound CT MRI 20:47-55.

43. Sathyanarayana S, Bottomley PA (2009) MRI endoscopy using intrinsically localized probes. Med Phys 36:908-919.

44. Zagoria RJ, Schlarb CA, Ott DJ, Bechtold RE, Wolfman NT, Scharling ES, Chen MYM, Loggie BW (1997) Assessment of rectal tumor infiltration utilizing endorectal MR imaging and comparison with endoscopic rectal sonography. J Surg Oncol 64:312-317.

45. D'Amico AV, Schnall M, Whittington R, Malkowicz SB, Schultz D, Tomaszewski JE, Wein A (1998) Endorectal coil magnetic resonance imaging identifies locally advanced prostate cancer in select patients with clinically localized disease. Urology 51:449-454.

46. Verret JM, Rabrait C, Pilleul F, Beuf O (2012) Réalisation de capteurs endoluminaux en Imagerie de Résonance Magnétique à 3T: performances et sécurité. Proc. 1st Meet. Société Fr. Résonance Magnétique En Biol. Médecine. Marseille, $\mathrm{p} 1$

47. Ayde R, Gaborit G, Jarrige P, Duvillaret L, Sablong R, Perrier A, Beuf O (2013) Potentialities of an Electro-Optic Crystal Fed by Nuclear Magnetic Resonant Coil for Remote and Low-Invasive Magnetic Field Characterization. IEEE Sens J 13:1274-1280. 
656
48. Fandrey S, Weiss S, Muller J (2008) Development of an Active Intravascular MR Device With an Optical Transmission System. IEEE Trans Med Imaging 27:1723-1727.

49. Syms R, Solymar L, Young IR (2010) Periodic Analysis of MR-Safe Transmission Lines. IEEE J Sel Top Quantum Electron 16:433-440.

50. Thörmer G, Reiss-Zimmermann M, Otto J, Hoffmann K-T, Moche M, Garnov N, Kahn T, Busse H (2013) Novel technique for MR elastography of the prostate using a modified standard endorectal coil as actuator. J Magn Reson Imaging 37:1480-1485.

51. Larsson AE, Melgar S, Rehnström E, Michaëlsson E, Svensson L, Hockings P, Olsson LE (2006) Magnetic resonance imaging of experimental mouse colitis and association with inflammatory activity. Inflamm Bowel Dis 12:478-485.

52. Yeung CJ, Susil RC, Atalar E (2002) RF safety of wires in interventional MRI: using a safety index. Magn Reson Med 47:187-193.

53. Konings MK, Bartels LW, Smits HFM, Bakker CJG (2000) Heating Around Intravascular Guidewires by Resonating RF Waves. J Magn Reson Imaging 12:79-85. 

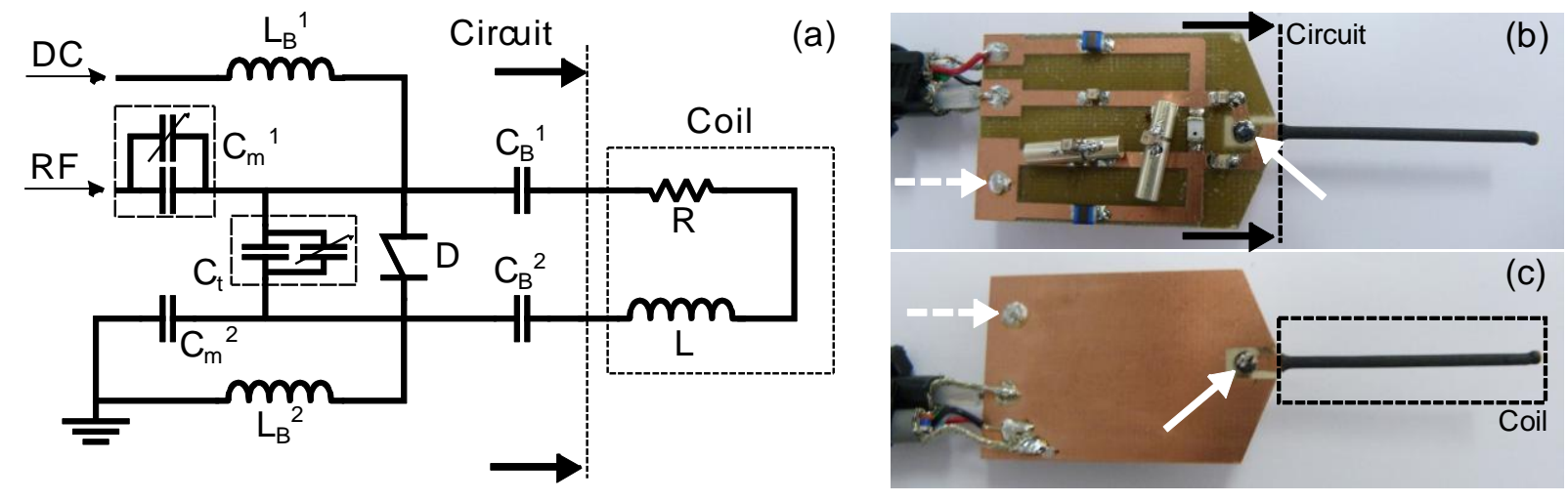

Legend of Fig1:

661 Fig 1 (a) Electric scheme of the coil. The corresponding part between the scheme and the pictures are

662 illustrated. (b) The upper face of the coil with the soldered components constitutes the RLC resonator.

663 On (c) is represented the lower face of the prototype. Straight white arrow is the location of the plated

664 drill between the two faces for connecting the electrical circuit to the coil. White dashed arrow is the 665 location of the connection between the grounds (DC and RF) to the ground plane.Values of 666 components are mentioned in Table 1 


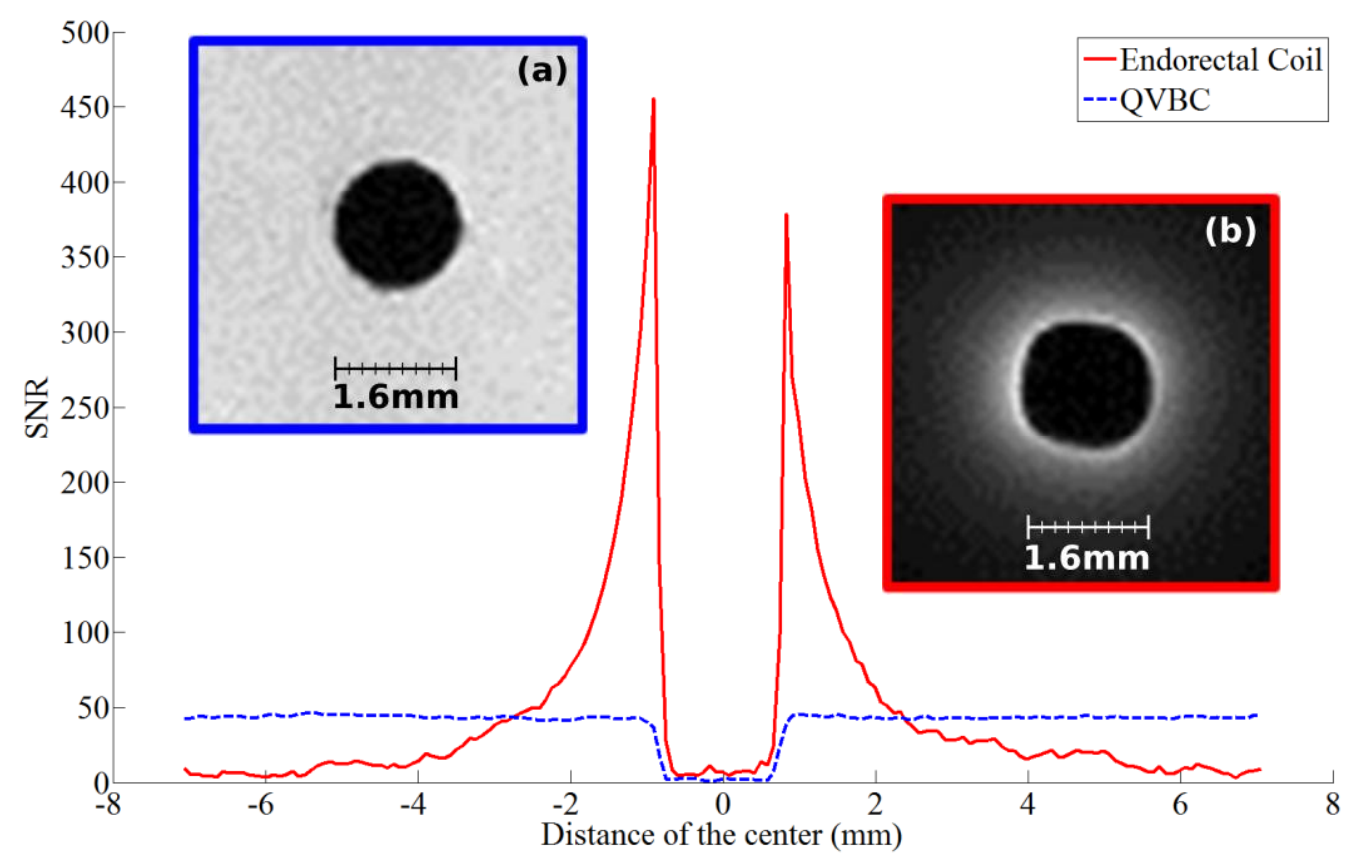

669 Legend of Fig 2:

670 Fig 2 Comparison of the EC SNR profile (straight profile) and the QVBC SNR profile (dashed profile)

671 obtained on a phantom containing $1.25 \mathrm{~g} / \mathrm{L} \mathrm{NiSO} 4$ and $5 \mathrm{~g} / \mathrm{L} \mathrm{NaCl}$. (a) is the image used for plotting

672 the QVBC profile and (b) the image used for the EC profile. Close to the coil the SNR is drastically

673 better than the one obtained with a dedicated quadrature birdcage coil

674 

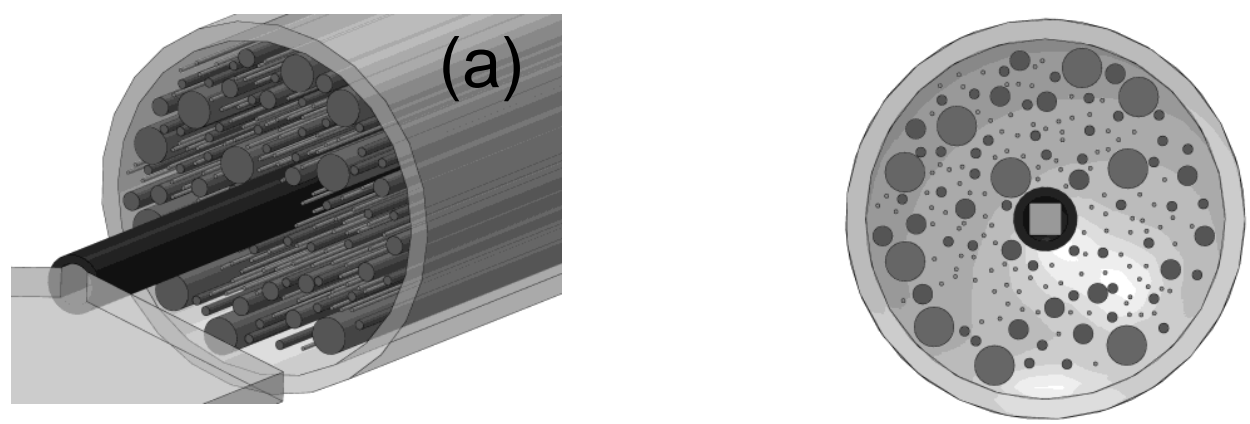

(b)
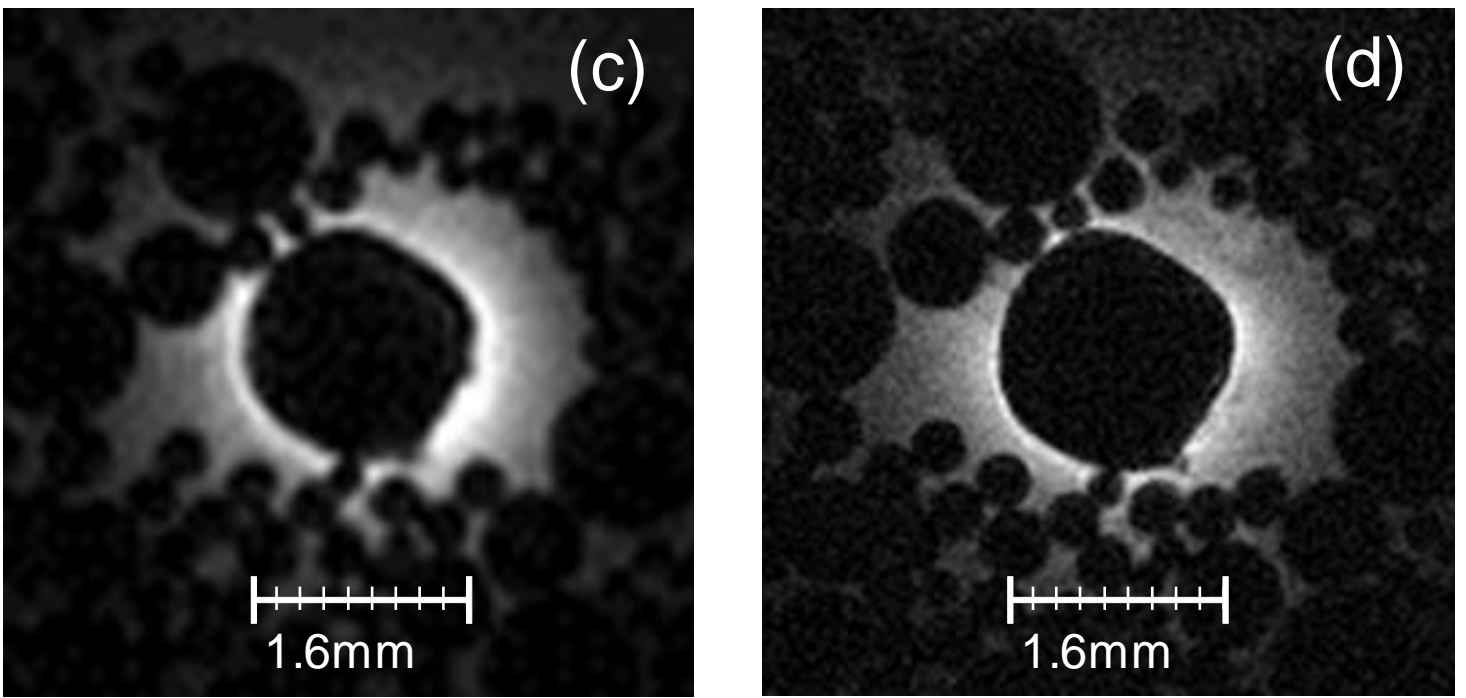

675

Legend of Fig 3:

677 Fig 3 Images of the phantom created for the purpose of the experiments. (a) and (b) represent a 678 reconstruction of the phantom containing optical fibers of different diameters. (c) and (d) are the 679 corresponding MR images obtain with FLASH sequences at different voxel size (50x50 $\mu \mathrm{m}^{2}$ for (c) and 680 $\left.39 \times 39 \mu \mathrm{m}^{2}(\mathrm{~d})\right)$. The slice thickness was kept constant and equals to $234 \mu \mathrm{m}$ 

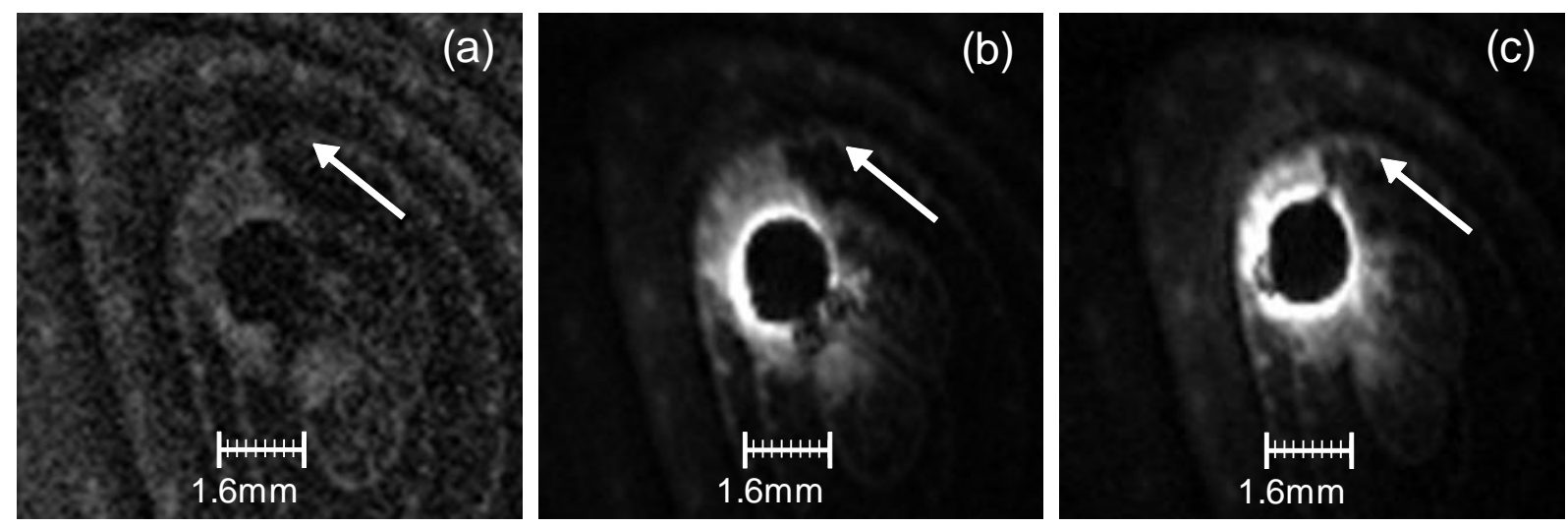

683

Legend of Fig 4:

684

Fig 4 MR images of onion. (a) the volume coil was set in E/R mode, the lamellar structure represented

by the white arrow are not correctly distinguishable. Switching the configuration by setting the VC in emission mode and the EC in reception mode the SNR and image quality are improved. On (b) the 
692

693

694

695

696

697

698

699

700
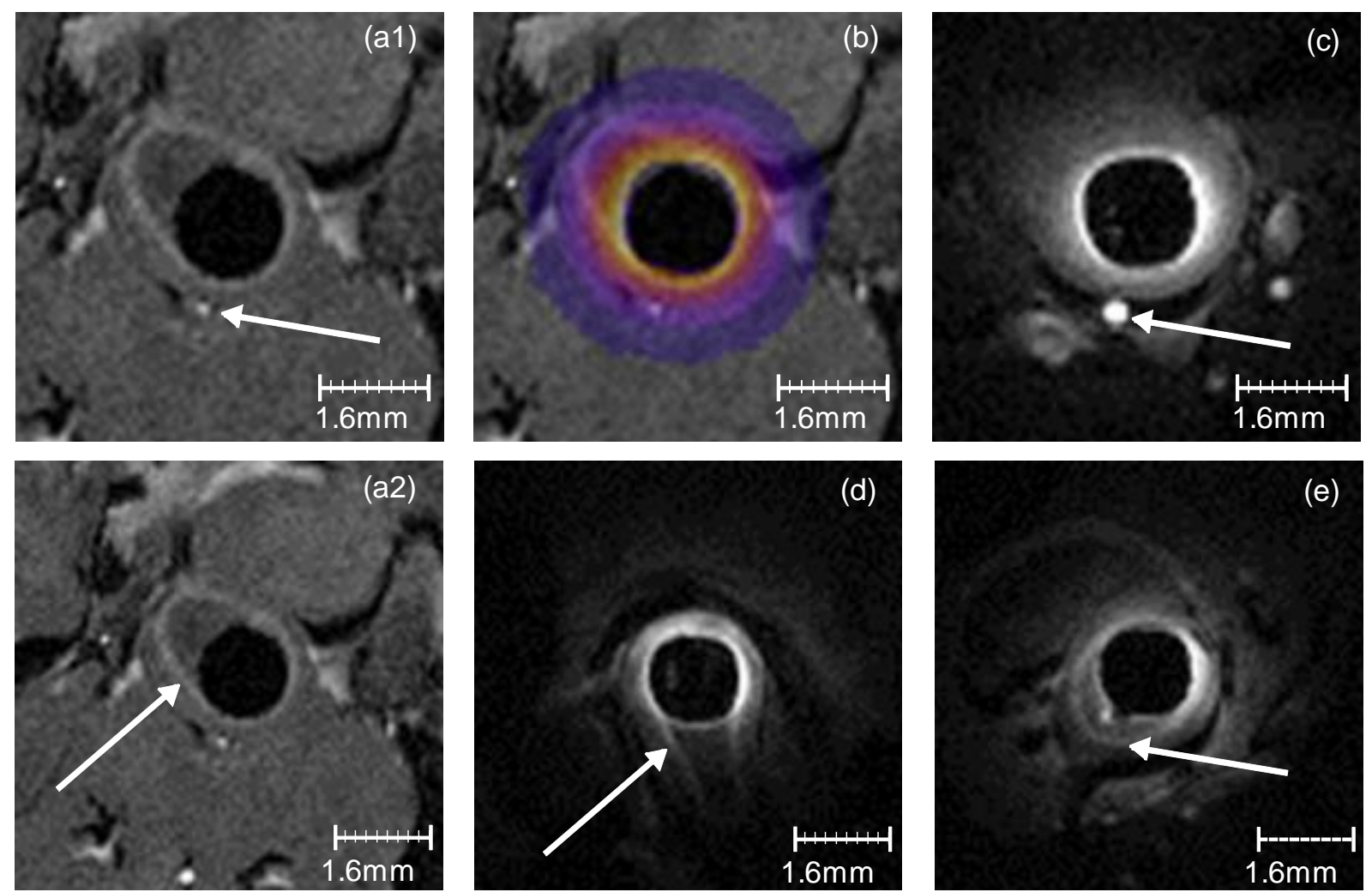

Legend of Fig 5:

Fig 5 in vivo experiments were performed on a mouse model of colitis. MR images acquired with the QVBC show blood vessels on (a1) and the colon wall on (a2) - white arrows. The SNR is too low to correctly distinguish the colon wall layers in details. The use of the EC allows to increase the local SNR. On (b) is represented the ratio between the SNR ratio of the QVBC and the EC. The EC FOV is sufficient for the region of interest. On (c), white arrow locates the same blood vessel than on (a1) and it is used for colocalization. (d) and (e) are showing the colon wall layer on different slices 

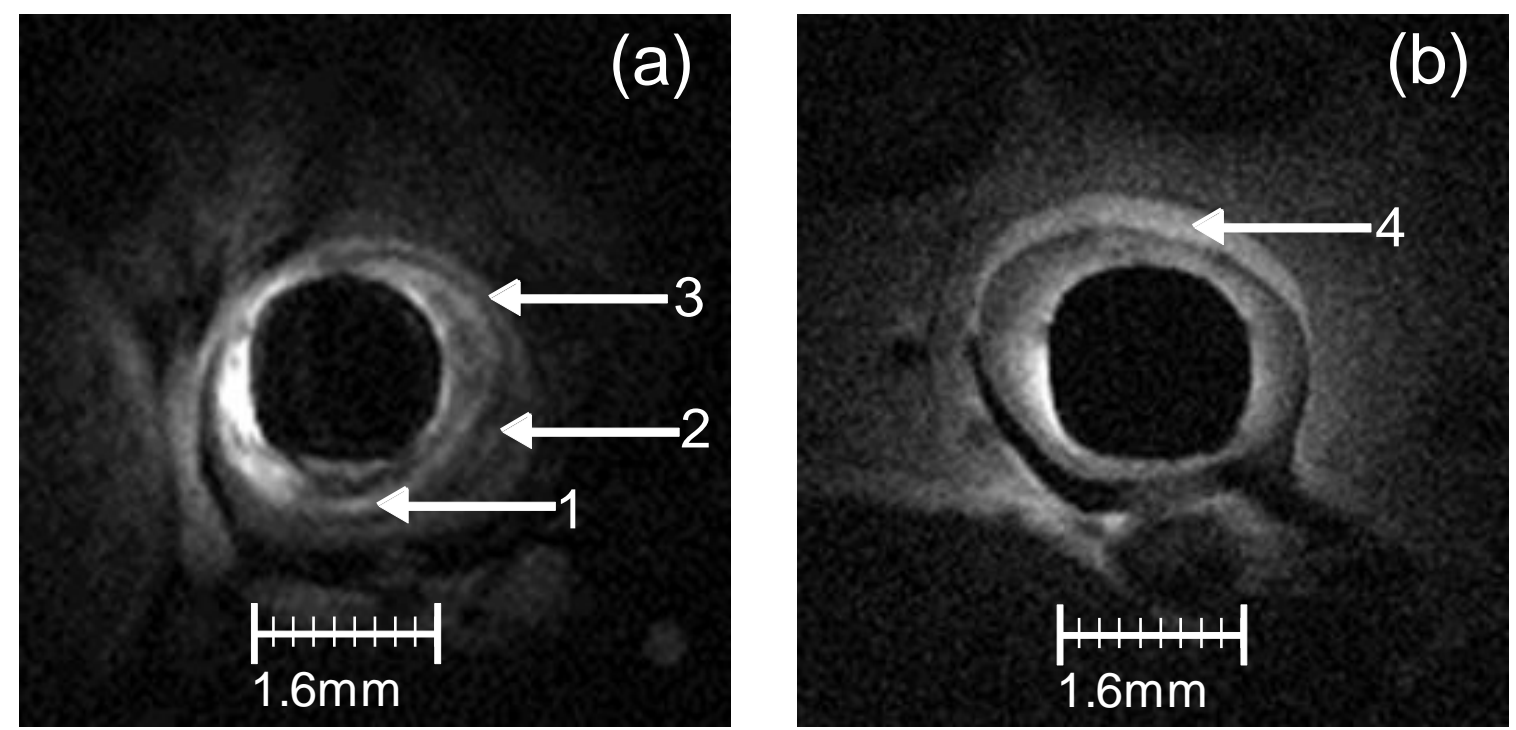

Legend of Fig 6:

704 Fig 6 From (a) to (b) the spatial resolution was increased up to $39 \times 39 \mu \mathrm{m}^{2}$ (slice thickness of $234 \mu \mathrm{m}$ ).

705 It is then possible to depict the colon wall complex in details. (1) refers to the mucosa, (2) to the submucosa and (3) to the muscularis propria. (4) Represents a thin muscle layer closed to the coil. On

(b) the colon wall seems to have disappeared 

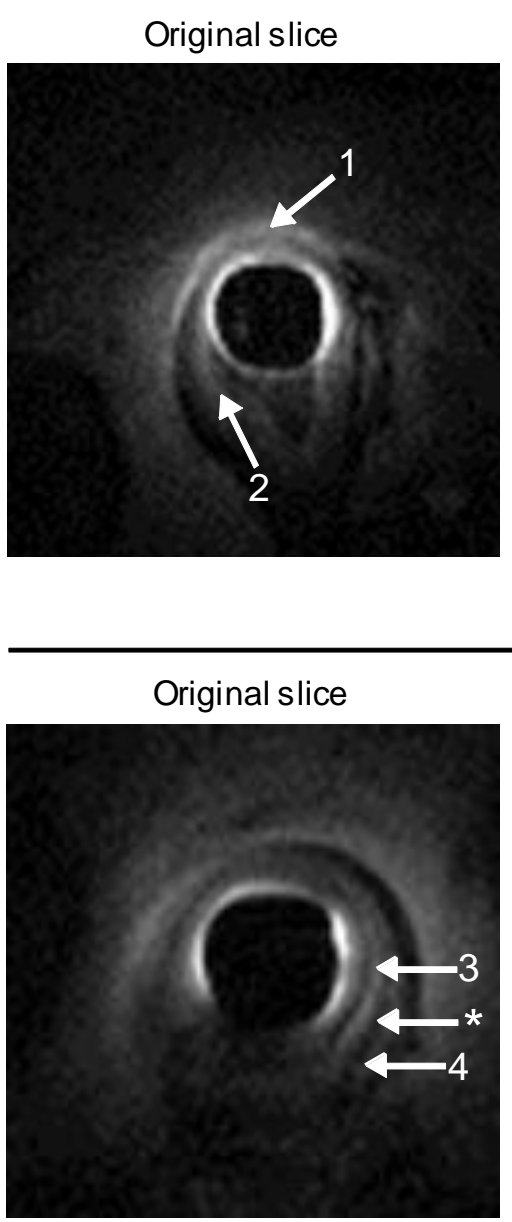

710

711

712

713

714

715

716

Legend of Fig 7: previous one and show a very different $\mathrm{T} 2$ time

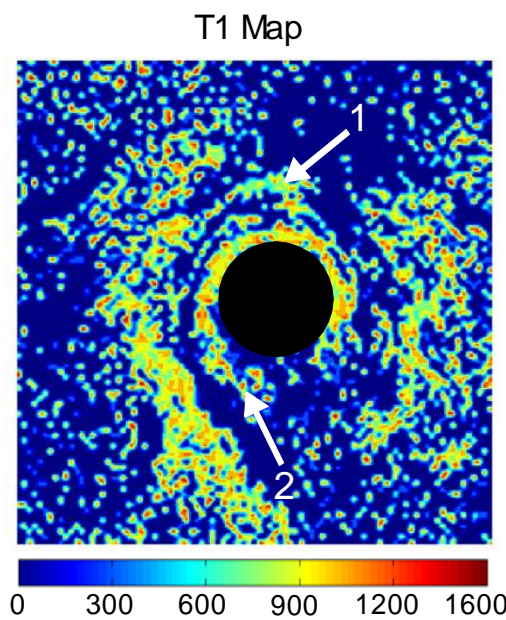

T1 Map Fit quality $\left(r^{2}\right)$

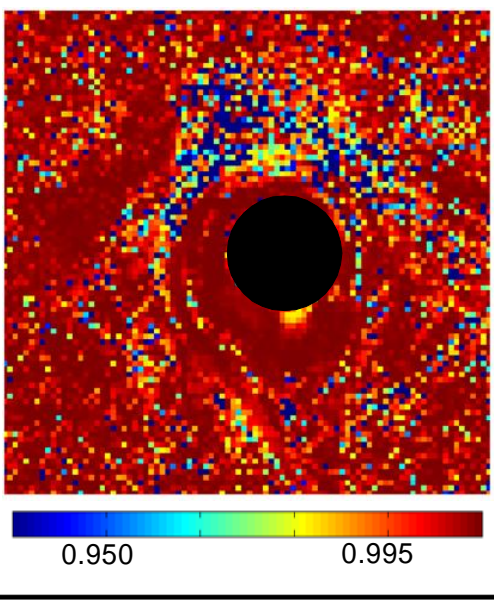

T2 Map Fit quality $\left(r^{2}\right)$
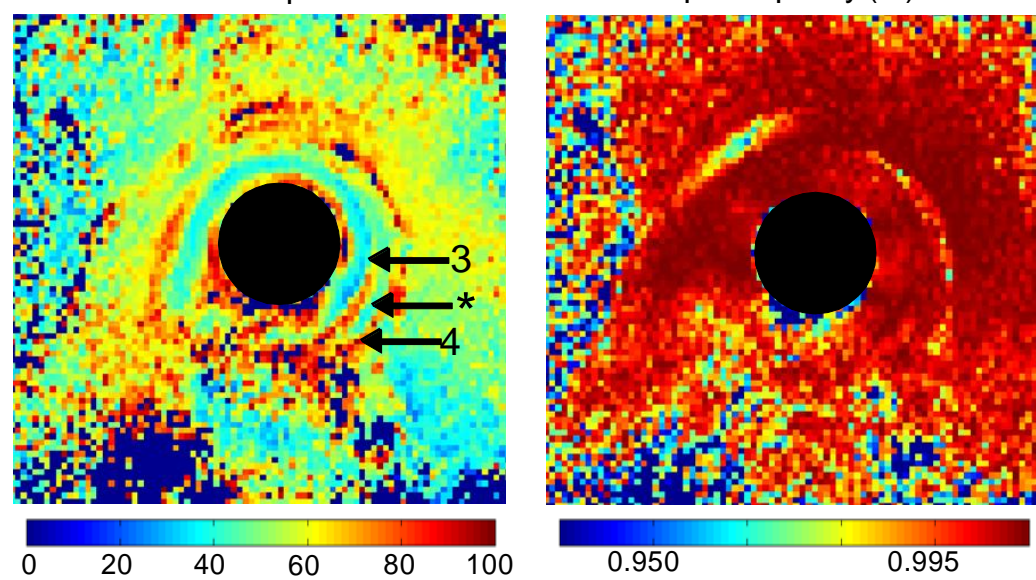

Fig 7 T1- and T2-maps obtained on different slices. (1) indicates the location of a thin muscle structure having T1 relaxation times lower than the ones observed on the colon wall (2). (3) and (4) represents two different layers (mucosa and muscularis propria) which nearly identical physiological constitutions so on with their respective $\mathrm{T} 2$ relaxation times. (*) locates the layer between the two 
Table 1 Components used to build the endoluminal coil with values, manufacturers and references.

\begin{tabular}{|c|c|c|c|}
\hline Function & Name & Value & Components \\
\hline \multirow[t]{2}{*}{ Tuning } & \multirow[t]{2}{*}{$\mathbf{C t}$} & $22 p F+1 p F$ & Non-variable non-magnetic capacitor \\
\hline & & from $0.8 \mathrm{pF}$ to $8 \mathrm{pF}$ & Non-magnetic trimmer capacitor \\
\hline \multirow[t]{3}{*}{ Matching } & \multirow[t]{2}{*}{ Cm1 } & $5.6 \mathrm{pF}$ & Non-variable non-magnetic case A capacitor \\
\hline & & from $0.8 \mathrm{pF}$ to $8 \mathrm{pF}$ & Non-magnetic trimmer capacitor \\
\hline & $\mathrm{Cm} 2$ & $5.6 \mathrm{pF}+2.2 \mathrm{pF}+1 \mathrm{pF}$ & Non-variable non-magnetic case A capacitor \\
\hline \multirow[t]{2}{*}{ Blocking } & Cb1 & $100 \mathrm{pF}+82 \mathrm{pF}$ & Non-variable non-magnetic case A capacitor \\
\hline & $\mathbf{C b 2}$ & $100 \mathrm{pF}+82 \mathrm{pF}$ & Non-variable non-magnetic case A capacitor \\
\hline \multirow[t]{3}{*}{ Active decoupling } & D & $\mathbf{x}$ & PIN diode \\
\hline & LB1 & $1.2 \mu \mathrm{H}$ & Choke inductors \\
\hline & LB2 & $1.2 \mu \mathrm{H}$ & Choke inductors \\
\hline
\end{tabular}


Table 2 Sequence and parameters used for the in vivo protocol. The scout sequences are not mentioned.

\begin{tabular}{|c|c|c|c|c|c|c|c|c|c|}
\hline Sequence & Orientation & $\begin{array}{c}\text { FOV } \\
\left(\mathrm{mm}^{2}\right)\end{array}$ & $\begin{array}{c}\text { Slab/Slice } \\
\text { Thickness } \\
(\mathrm{mm})\end{array}$ & $\begin{array}{c}\text { TR/TE } \\
(\mathrm{ms} / \mathrm{ms})\end{array}$ & $\begin{array}{c}\text { Flip Angle } \\
\left({ }^{\circ}\right)\end{array}$ & Matrix & $\begin{array}{c}\text { Voxel Size } \\
\left(\mu \mathrm{m}^{3}\right)\end{array}$ & $\begin{array}{c}\text { Receive } \\
\text { BW }(\mathrm{kHz})\end{array}$ & $\begin{array}{c}\text { Acquisition } \\
\text { Time }\end{array}$ \\
\hline Flash 3D & Axial & $16 \times 16$ & $1 \times 15$ & $20 / 6.653$ & $15 / 20 / 25$ & $192 \times 192 \times 64$ & $83 \times 83 \times 234$ & 25 & $8: 11^{\prime}$ \\
\hline FLASH 3D & Coronal & $32 \times 32$ & $1 \times 5$ & $23.87 / 8.19$ & 20 & $384 \times 384 \times 16$ & $83 \times 83 \times 312$ & 25 & $2: 26^{\prime}$ \\
\hline Multi TE & axial & $10 \times 10$ & $6 \times 1.5$ & $2000 / 12,8+6 \times 12,6$ & $/$ & $128 \times 128 \times 66$ & $78 \times 78 \times 308$ & 25 & $6: 24^{\prime}$ \\
\hline
\end{tabular}


Table 3Sequences used for the characterization of the $\mathrm{EC}$ and the assessment of the $\mathrm{EC}$ during in vitro experiments.

\begin{tabular}{|c|c|c|c|c|c|c|c|c|c|}
\hline Sequence & Orientation & $\begin{array}{c}\text { FOV } \\
\left(\mathrm{mm}^{2}\right)\end{array}$ & $\begin{array}{c}\text { Slab Thickness } \\
(\mathrm{mm})\end{array}$ & $\begin{array}{c}\text { TR/TE } \\
(\mathrm{ms} / \mathrm{ms})\end{array}$ & $\begin{array}{c}\text { Flip Angle } \\
\left({ }^{\circ}\right)\end{array}$ & Matrix & $\begin{array}{c}\text { Voxel Size } \\
\left(\mu \mathrm{m}^{3}\right)\end{array}$ & $\begin{array}{c}\text { Receive } \\
\text { BW (kHz) }\end{array}$ & $\begin{array}{c}\text { Acquisition } \\
\text { Time }\end{array}$ \\
\hline Flash 3D & Axial & $16 \times 16$ & $1 \times 15$ & $20 / 6.653$ & 20 & $192 \times 192 \times 64$ & $83 \times 83 \times 234$ & 25 & $8: 11^{\prime}$ \\
\hline FLASH 3D & axial & $10 \times 10$ & $1 \times 15$ & $20 / 6.1$ & 20 & $256 \times 256 \times 64$ & $39 \times 39 \times 234$ & 25 & $10: 55^{\prime}$ \\
\hline
\end{tabular}

